\title{
Rotation of surviving companion stars after type la supernova explosions in the WD+MS scenario
}

\author{
Z.-W. Liu ${ }^{1,2,3,4}$, R. Pakmor ${ }^{5,4}$, F. K. Röpke ${ }^{6,4}$, P. Edelmann ${ }^{4}$, W. Hillebrandt ${ }^{4}$, W. E. Kerzendorf ${ }^{7,8}$, \\ B. Wang ${ }^{1,2}$, and Z. W. Han ${ }^{1,2}$ \\ ${ }^{1}$ National Astronomical Observatories/Yunnan Observatory, Chinese Academy of Sciences, Kunming 650011, PR China \\ e-mail: zwliu@ynao.ac.cn \\ 2 Key Laboratory for the Structure and Evolution of Celestial Objects, Chinese Academy of Sciences, Kunming 650011, PR China \\ 3 University of Chinese Academy of Sciences, Beijing 100049, PR China \\ ${ }^{4}$ Max-Planck-Institute für Astrophysik, Karl-Schwarzschild-Str. 1, 85741 Garching, Germany \\ 5 Heidelberger Institut für Theoretische Studien, Schloss-Wolfsbrunnenweg 35, 69118 Heidelberg, Germany \\ ${ }^{6}$ Institut für Theoretische Physik und Astrophysik, Universität Würzburg, Am Hubland, 97074 Würzburg, Germany \\ 7 Research School of Astronomy and Astrophysics, Mount Stromlo Observatory, Cotter Road, Weston Creek, ACT 2611, Australia \\ 8 Department of Astronomy and Astrophysics, University of Toronto, 50 Saint George Street, Toronto, ON M5S 3H4, Canada
}

Received 13 December 2012 / Accepted 5 March 2013

\begin{abstract}
Context. In the single-degenerate (SD) scenario of type Ia supernovae (SNe Ia) the non-degenerate companion star survives the supernova (SN) explosion and thus should be visible near the center of the SN remnant and may show some unusual features. Therefore, a promising approach to test progenitor models of SNe Ia is to search for the companion star in historical SN remnants. Aims. Here we present the results of three-dimensional (3D) hydrodynamics simulations of the interaction between the SN Ia blast wave and a main-sequence companion taking into consideration its orbital motion and spin. The primary goal of this work is to investigate the rotation of surviving companion stars after SN Ia explosions in the WD+MS scenario.

Methods. We used Eggleton's stellar evolution code including the optically thick accretion wind model to obtain realistic models of companion stars. The impact of the supernova blast wave on these companion stars was followed in 3D hydrodynamic simulations employing the smoothed particle hydrodynamics (SPH) code GADGET3.

Results. We find that the rotation of the companion star does not significantly affect the amount of stripped mass and the kick velocity caused by the SN impact. However, in our simulations, the rotational velocity of the companion star is significantly reduced to about $14 \%$ to $32 \%$ of its pre-explosion value due to the expansion of the companion and because $55 \%-89 \%$ of the initial angular momentum is carried away by the stripped matter.

Conclusions. Compared with the observed rotational velocity of the presumed companion star of Tycho's supernova, Tycho G, of $\sim 6 \mathrm{~km} \mathrm{~s}^{-1}$, the final rotational velocity we obtain in our simulations is still higher by at least a factor of two. Whether this difference is significant and may cast doubts on the suggestion that Tycho G is the companion of SN 1572 has to be investigated in future studies. Based on binary population synthesis results, we present for the first time the expected distribution of rotational velocities of companion stars after the SN explosion, which may provide useful information for the identification of the surviving companion in observational searches in other historical SN remnants.
\end{abstract}

Key words. stars: rotation - supernovae: general - hydrodynamics - binaries: close

\section{Introduction}

Type Ia SNe (SNe Ia) are used as cosmic distance indicators because their luminosity can be calibrated based on the empirical relation between light curve shape and peak luminosity (Phillips 1993; Phillips et al. 1999). This has provided the first evidence for the accelerating expansion of the present Universe (Riess et al. 1998; Perlmutter et al. 1999; Leibundgut 2008). SNe Ia are also believed to be important contributors to the cosmic nucleosynthesis, and they are sources of kinetic energy in evolution process of galaxies. Although a detailed understanding of the nature of their progenitors and the physics of explosion is still lacking (see Hillebrandt \& Niemeyer 2000 for a review), there is consensus that $\mathrm{SNe}$ Ia arise from thermonuclear explosions of carbon/oxygen white dwarfs (CO WDs) in binary systems (Hoyle \& Fowler 1960; Nomoto et al. 1997).
In principle, there are various possibilities for the evolution toward an explosion (see Wang \& Han 2012 for a review). One option is a merger of two CO WDs with a combined mass in excess of the Chandrasekhar mass, which may explode as an SN Ia, the double-degenerate (DD) scenario (Iben \& Tutukov 1984; Webbink 1984). The DD model can explain in a natural way the lack of hydrogen in SNe Ia. However, only a few DD systems have been found whose orbital period is short enough that they will merge within a Hubble time, but in none of them the combined mass exceeds the Chandrasekhar mass limit. On the other hand, in recent numerical simulations of Pakmor et al. $(2010,2011)$ it was found that the violent merger of a pair of white dwarfs with equal masses of $\sim 0.9 M_{\odot}$ can directly trigger a thermonuclear explosion that resembles subluminous 1991bglike SNe Ia. Moreover, it was shown that the violent merger of two CO WDs with masses of $0.9 M_{\odot}$ and $1.1 M_{\odot}$ produces 
lightcurves and spectra that agree well with those of normal SNe Ia (Pakmor et al. 2012b), supporting the DD scenario.

On the other hand, a rather massive WD may accrete hydrogen-rich material from a non-degenerate binary companion until it approaches the Chandrasekhar mass, the singledegenerate (SD) scenario. In this case, the binary companion could be a main-sequence (MS) star (WD+MS channel), a slightly evolved subgiant star, or a red-giant (RG) star (Han \& Podsiadlowski 2004; Wang \& Han 2010b). The lack of hydrogen in observed SN Ia spectra can be seen as troublesome for the SD scenario because the companions are hydrogenrich stars. With more realistic MS companion star models than those used in previous work, Liu et al. (2012) performed threedimensional (3D) smooth particle hydrodynamics (SPH) simulations of the interaction between SN Ia ejecta and the MS companion star. The authors found that in all cases they considered, more than $0.1 M_{\odot}$ was stripped from the hydrogen-rich companion and was mixed into the SN ejecta, which disagrees with most recent observational constraints on the presence of hydrogen in SN Ia from nebular spectra $\sim 0.01 M_{\odot}$ (Leonard 2007; Shappee et al. 2013). Moreover, no other similar hydrodynamics simulations showed an amount of stripped mass below this strong observational limit (see Marietta et al. 2000; Pakmor et al. 2008; Pan et al. 2010; Ricker et al. 2010; Pan et al. 2012b). On the other hand, there is evidence from observations that the progenitors of at least some SNe Ia come from the SD channel. For instance, evidence for circumstellar matter and features indicative for an interaction between the SN and circumstellar matter were found recently (Patat et al. 2007; Sternberg et al. 2011; Foley et al. 2012; Dilday et al. 2012).

The merger of two WDs leaves no remnant after an SN Ia explosion. In contrast, in the SD scenario the companion star survives the explosion and, in principle, can be identified due to its peculiar spatial velocity, its rotation, effective temperature, luminosity, or composition (Han 2008; Wang \& Han 2010a). Therefore, it is a promising approach to test the progenitors of $\mathrm{SNe}$ Ia by directly searching for the surviving companion star in galactic SN remnants (SNRs). There are several ways in which the SN blast wave modifies the properties of the companion. First, the SN strips off matter from the surface of the companion and injects thermal energy into it during the interaction. This causes the surviving companion to expand and lowers its surface gravity. Secondly, after the impact the companion star's surface will be enriched with heavy elements (e.g., Ni, Fe or Ca) from the inner part of the SN ejecta, which should be detectable in its spectrum (see González Hernández et al. 2009; Pan et al. 2012a,b). Finally, the companion star retains its preexplosion orbital velocity after the explosion, which gives it a peculiar velocity compared to other stars in the vicinity.

Tycho Brahe's SN 1572 is an SN Ia that exploded in the Milky Way. Ruiz-Lapuente et al. (2004) have analyzed the stars within a circle of 0.65 arcmin radius of the center of the SNR up to an apparent visual magnitude $V=22$. They found a star, Tycho G, similar to the Sun in surface temperature and luminosity, but with a lower surface gravity than an MS star. It has a significant peculiar velocity in radial and proper motion and moves at more than three times the mean velocity of the other stars in the field. Therefore, they suggested that Tycho G star could be the surviving companion star of SN 1572. However, since then it has been noted that Tycho $\mathrm{G}$ does not show any spectral peculiarities (Ihara et al. 2007) and that it is apparently not out of thermal equilibrium (Howell 2011). Fuhrmann (2005) also claimed that it might be a thick-disk star coincidentally passing in the vicinity of the remnant of SN 1572. Recently, González Hernández et al. (2009) found that Tycho G has an overabundance of Ni relative to normal metal-rich stars, and they suggested that Tycho $\mathrm{G}$ could have captured the low-velocity tail of the SN 1572 ejecta, which upholds Tycho $\mathrm{G}$ as a surviving companion star again. However, the measured $[\mathrm{Ni} / \mathrm{Fe}]$ ratio from a more recent study of Kerzendorf et al. (2012) seems to be not so unusual with respect to field stars with the same metallicity.

Because of the strong tidal coupling of a Roche-lobe filling donor in the SD case, the donor star rotation is expected to be tidally locked to its orbital motion. This forces the binary donor star to have a spin corresponding to the orbital frequency of the binary system (Han \& Podsiadlowski 2004; Kerzendorf et al. 2009). After the SN explosion, the companion is released from its orbit and continues to rotate. Therefore, fast post-explosion rotation might be a signature of the donor star. The survivor could be in rapid rotation, which would be easily measurable (see Kerzendorf et al. 2009, 2012). With the HIRES instrument on the Keck-I telescope and with Subaru high-resolution spectroscopy of star G in the Tycho SNR, Kerzendorf et al. (2009, 2012) measured the rotational velocity of Tycho $G$ and obtained only $\sim 6 \pm 1.5 \mathrm{~km} \mathrm{~s}^{-1}$. Thus, they concluded that Tycho $\mathrm{G}$ is unlikely to be the surviving companion star of SN 1572 because it does not have the expected high rotational velocity (Kerzendorf et al. 2009; Howell 2011). However, the impact of the SN ejecta strips off material from the surface of the companion star, which can significantly reduce its angular momentum, thereby lowering the rotational velocity. Moreover, the $\mathrm{SN}$ impact and heating bloat the companion star, which will also slow down its spin. Recently, Pan et al. (2012a) computed the post-impact evolution of the remnant star produced in their multi-dimensional adaptive mesh refinement simulations of WD+MS-like models with the FLASH code (Pan et al. 2012b). In line with the arguments given above, they concluded that Tycho $\mathrm{G}$ cannot be eliminated as being a promising progenitor candidate based on its low rotational surface velocity only (Pan et al. 2012a).

In this paper, we present the results of 3D SPH simulations of the impact of SN Ia ejecta on an MS companion star including the orbital motion and spin of the companion. The purpose of the work is to investigate how the SN Ia impact changes the rotation rate of the companion, which will be useful for identifying the surviving companion star in SNRs. The paper is organized as follows. In Sect. 2, we describe the codes used and show an example of the initial companion star models used in our simulations. In Sect. 3 the effect of rotation on the interaction between SN Ia ejecta and its MS companion are discussed. Post-impact rotation features of an MS star are shown in detail in Sect. 4. Furthermore, the results of our simulations are compared with the observed rotation of star Tycho G in Sect. 5. Next in Sect. 6, we discuss the distribution of the rotational velocities of the remnant stars after thermal equilibrium is reestablished. Finally, in Sect. 7, we summarize our results.

\section{Numerical method and model}

\subsection{Numerical codes and initial setup}

To obtain consistent MS companion star models at the onset of the SN explosion, similar to Liu et al. (2012), we used Eggleton's one-dimensional (1D) stellar evolution code (Eggleton 1971, $1972,1973)$ to follow the binary evolution of an SD progenitor system in detail. Roche-lobe overflow (RLOF) is treated in the code as described by Han \& Podsiadlowski (2004). The 
opacity tables used in our calculations were compiled by Chen \& Tout (2007) from Iglesias \& Rogers (1996) and Alexander \& Ferguson (1994). We used a typical population I composition with hydrogen abundance $X=0.70$, helium abundance $Y=0.28$, and metallicity $Z=0.02$. We set $\alpha=l / H_{\mathrm{P}}$, the ratio of mixing length to the local pressure scale height, to 2 and set the convective overshooting parameter $\delta_{\text {ov }}$ to 0.12 (Pols et al. 1997; Schroder et al. 1997), which roughly corresponds to an overshooting length of $\sim 0.25$ pressure scale height.

Furthermore, the latest version of the SPH code GADGET-3 (Springel et al. 2001; Springel 2005) was employed to simulate the impact of SN Ia explosions on their MS companions by including the orbital and spin velocities of companion stars. The GADGET code was originally used for cosmological simulations, but it has been modified to make it applicable to stellar astrophysics problems (see Pakmor et al. 2012a) and it has been used successfully to capture the main dynamical effects of the SN impact on its companion star (see Pakmor et al. 2008; Liu et al. 2012). In our simulations, we aim at determining the rotational velocity of the companion star after the impact. Therefore the fact that no matter leaves the computational domain and that momentum, energy, and angular momentum are strictly conserved are a crucial advantage of SPH over grid-based methods for the problem under investigation.

In this work, the basic setup for the GADGET code is almost the same as in Liu et al. (2012). The smoothing length was chosen such that a sphere of its radius encloses 60 neighboring particles. All particles have the same mass. The gravitational softening length is equal to the smoothing length. To reduce numerical noise introduced by mapping the initial model, the MS companion star was relaxed for $1.0 \times 10^{4} \mathrm{~s}$ (several dynamical timescales) before we started the actual impact simulation. The W7 SN Ia model (Nomoto et al. 1984) was used for the SN explosion, and the orbital separation was taken from the 1D consistent binary-evolution calculations.

Additionally, we assumed that the companion star co-rotates with its orbit due to strong tidal interaction. Thus the spin period of the companion star is locked with its orbital period. Furthermore, the orbital and spin velocities of the companion stars were included in our impact simulations to study the post-impact rotation rate of surviving star.

The system of coordinates was chosen as follows. We set the $x-y$ plane to be the orbital plane of the binary system and assumed a circular orbit. The $z$-axis was chosen as the spin axis of the companion, and the positive $z$-axis is the direction of the angular momentum. Finally, we assumed that the companion star rotates as a rigid body at the moment of the $\mathrm{SN}$ explosion.

\subsection{Progenitor model}

As in Liu et al. (2012) (see also Han \& Podsiadlowski 2004; Wang \& Han 2010a), we started our 1D consistent binary calculation after formation of the WD+MS system, the mass transfer then occurs through RLOF when the companion star fills its Roche lobe. Instead of solving the stellar equations for the WD star when we trace the detailed evolution of the companion star, the optically thick wind model of Hachisu et al. (1999) was adopted.

Figure 1 shows the evolution of a binary system with an initial donor-star mass of $M_{2}^{\mathrm{i}}=2.0 M_{\odot}$, an initial mass of the $\mathrm{CO} \mathrm{WD}$ of $M_{\mathrm{WD}}^{\mathrm{i}}=0.8 M_{\odot}$, and an initial orbital period of $\log \left(P^{\mathrm{i}} /\right.$ day $)=0.2$. In this system, the companion star has an orbital velocity of $138 \mathrm{~km} \mathrm{~s}^{-1}$ at the moment of the SN Ia
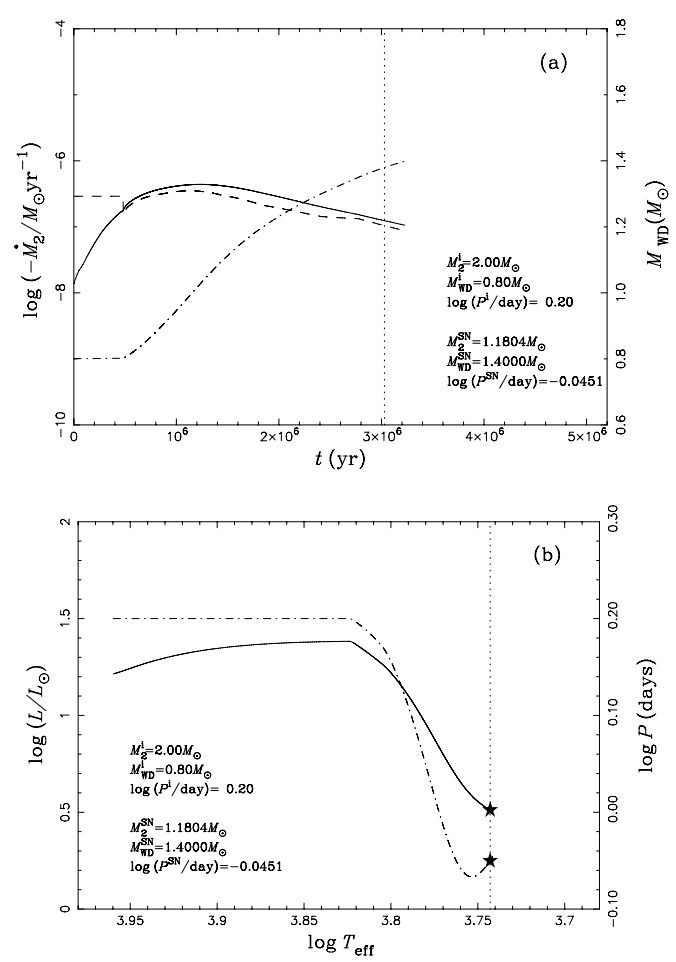

Fig. 1. Binary-evolution calculation of the MS_110 progenitor model. In panel a), the solid, dashed, and dash-dotted curves show the masstransfer rate, $\dot{M}_{2}$, the mass-growth rate of the CO WD, $\dot{M}_{\mathrm{CO}}$, and the mass of the CO WD, $M_{\mathrm{WD}}$. In panel $\mathbf{b}$ ), the evolutionary track of the donor star is shown as a solid curve and the evolution of the orbital period is shown as a dashed-dotted curve. The dotted vertical lines in both panels and asterisks in panel $\mathbf{b}$ ) indicate the position where the WD explodes as an SN Ia.

explosion. Assuming that the rotation of companion is locked with the orbital motion due to the strong tidal interaction, the rotational velocity $v_{\text {rot }}$ and its orbital velocity $v_{\text {orb,2 }}$ obey a simple relation as follows (see also Kerzendorf et al. 2009):

$v_{\mathrm{rot}}=\frac{M_{1}+M_{2}}{M_{1}} f(q) v_{\mathrm{orb}, 2}$,

where $q=M_{2} / M_{1}$ is the mass ratio of the binary system at the moment of the explosion, and $f(q)$ is the ratio of the Roche-lobe radius of companion star to the orbital separation (Eggleton 1973). Therefore, we calculate the rotational velocity of the companion star to be $v_{\text {rot }}^{\mathrm{SN}} \sim 110 \mathrm{~km} \mathrm{~s}^{-1}$ at the time of the explosion.

In this model, because hydrogen burning on the WD is unstable before the SN explosion, the system may be observed as a U Sco-type recurrent nova (Hachisu et al. 2008; Meng \& Yang 2010). With our consistent binary-evolution calculations, we obtain a CO WD+MS progenitor that is similar to the U Sco binary system with a mass of the secondary of $1.18 M_{\odot}$ and an orbital period $\sim 1$ day (see Table 1). This model is named "MS_110", the "MS" indicating a CO WD+MS binary system, the " 110 " means the rotational velocity of the companion star of $v_{\text {rot }}^{\mathrm{SN}} \sim 110 \mathrm{~km} \mathrm{~s}^{-1}$ at the moment of the explosion. U Scorpii is one of the bestobserved recurrent novae, and it has been suggested as a progenitor of an SN Ia because its white dwarf mass is close to the Chandrasekhar mass (Hachisu et al. 2000; Thoroughgood et al. 2001; Podsiadlowski 2003). To investigate the physical parameters of the recurrent nova, Hachisu et al. (2000) have successfully modeled the theoretical light curve for the outburst of the U Scorpii system with a model consisting of a WD mass of 
Table 1. SPH impact simulations for four different MS companion models.

\begin{tabular}{|c|c|c|c|c|c|c|c|c|c|c|}
\hline \multirow[t]{2}{*}{ Model name } & $M_{2}^{\mathrm{SN}}$ & $P^{\mathrm{SN}}$ & $R_{2}^{\mathrm{SN}}$ & $a^{\mathrm{SN}}$ & $v_{\mathrm{rot}}^{\mathrm{SN}}$ & $v_{\text {rot }}^{\mathrm{f}}$ & $v_{\mathrm{rot}}^{\mathrm{ff}}$ & $J_{\text {spin }}^{\mathrm{SN}}$ & $J_{\text {spin }}^{\mathrm{f}}$ & $M_{\text {bound }}$ \\
\hline & {$\left[M_{\odot}\right]$} & [days] & {$\left[R_{\odot}\right]$} & {$\left[R_{\odot}\right]$} & \multicolumn{3}{|c|}{$\left[\mathrm{km} \mathrm{s}^{-1}\right]$} & \multicolumn{2}{|c|}{$\left[10^{50} \mathrm{~g} \mathrm{~cm}^{2} \mathrm{~s}^{-1}\right]$} & {$\left[M_{\odot}\right]$} \\
\hline MS_160 & 1.21 & 0.29 & 0.93 & 2.55 & 160 & 52 & 98 & 2.94 & 1.31 & 1.04 \\
\hline MS_131 & 1.23 & 0.56 & 1.45 & 3.94 & 131 & 40 & 78 & 2.07 & 0.92 & 1.06 \\
\hline MS_110 & 1.18 & 0.91 & 1.97 & 5.39 & 110 & 25 & 46 & 2.25 & 0.62 & 0.95 \\
\hline MS_081 & 1.09 & 2.00 & 3.19 & 8.92 & 81 & 12 & 16 & 2.32 & 0.26 & 0.84 \\
\hline
\end{tabular}

Notes. Here, $M_{2}^{\mathrm{SN}}, P^{\mathrm{SN}}, R_{2}^{\mathrm{SN}}, a^{\mathrm{SN}}, v_{\mathrm{rot}}^{\mathrm{SN}}$, and $J_{\text {spin }}^{\mathrm{SN}}$ are the mass, the orbital period, the radius, the spin velocity, and the angular momentum of the companion star at the moment of the explosion. $v_{\mathrm{rot}}^{\mathrm{f}}, J_{\text {spin }}^{\mathrm{f}}$ and $M_{\text {bound }}$ denote the spin velocity, the angular momentum, and the total bound mass of the companion star after the SN impact. $v_{\text {rot }}^{\mathrm{ff}}$ is the rotational velocity at the surface after the thermal equilibrium is reestablished. Note that the rotational velocity $v_{\mathrm{rot}}^{\mathrm{SN}}$ is calculated by assuming that the rotation of the star is locked with the orbital motion due to tidal interactions.
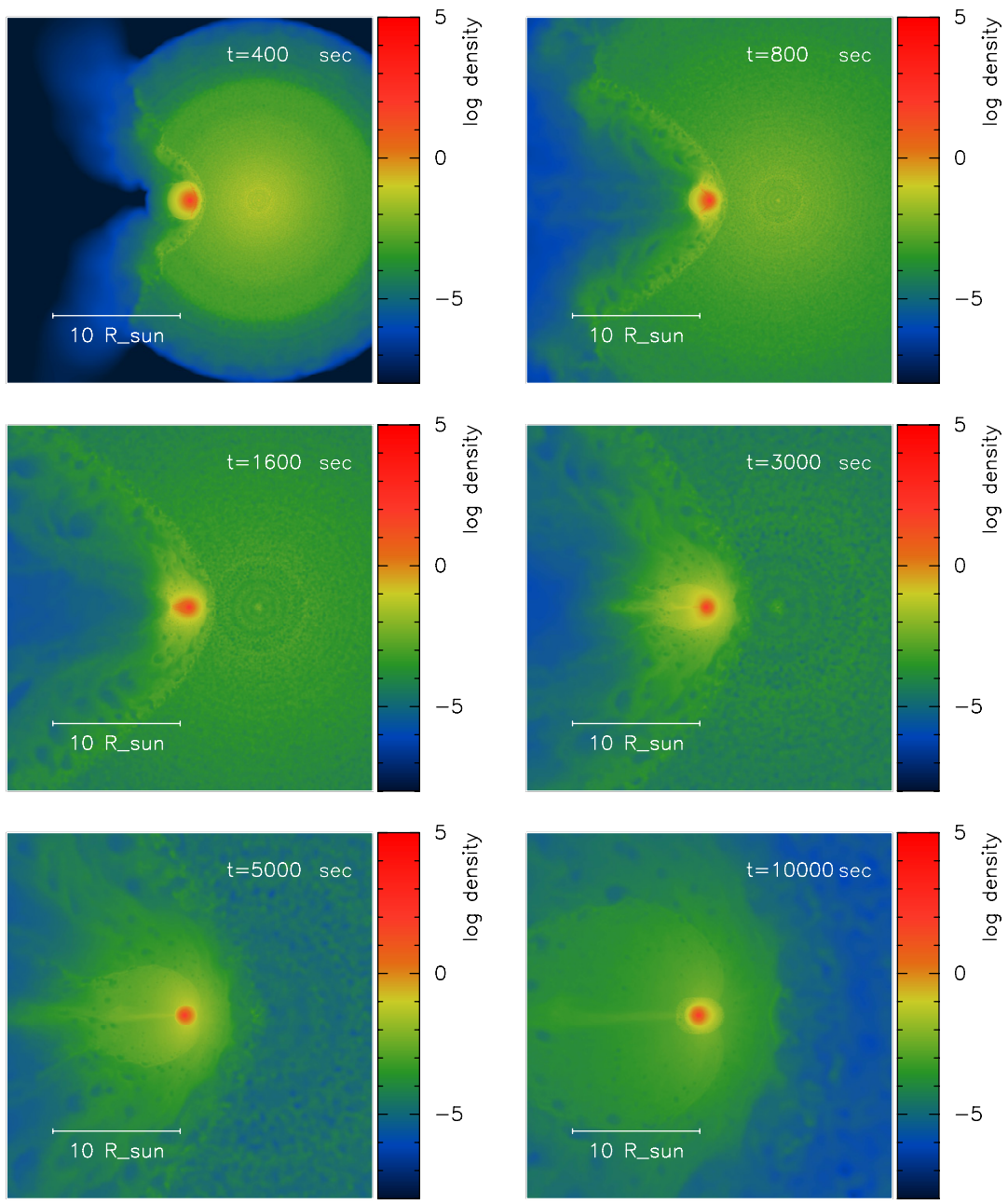

Fig. 2. Density distribution of the SN ejecta and the companion star of a non-rotating version of model MS_110. The color scale shows the logarithm of the density. The plots are made using the freely available SPLASH tool (Price 2007).

$M_{\mathrm{WD}}=1.37 M_{\odot}$, a secondary mass of $M_{2}=0.8-2.0 M_{\odot}$ and an orbital inclination of $\sim 80^{\circ}$.

\section{Effects of rotation}

Based on the system similar to U Sco (MS_110) that was discussed in Sect. 2.2, we performed a 3D SPH impact simulation that included the spin velocity of $v_{\text {rot }}^{\mathrm{SN}}=110 \mathrm{~km} \mathrm{~s}^{-1}$, and the orbital velocity of $v_{\mathrm{orb}, 2}^{\mathrm{SN}}=138 \mathrm{~km} \mathrm{~s}^{-1}$ to study the effect of asymmetry caused by the orbital motion and spin of the companion star. We used a total of $2 \times 10^{7}$ particles to represent the companion star only (which corresponds to a total number of particles in the simulation of $\sim 4 \times 10^{7}$ ). All particles had the same mass of $5.9 \times 10^{-8} M_{\odot}$. The SN properties were taken from the W7 explosion model (Nomoto et al. 1984) with an initial orbital separation of $3.75 \times 10^{11} \mathrm{~cm}$.

The temporal evolution of the spatial density distribution of both the companion and the SN ejecta MS_110 model with and without including the orbital motion and the rotational velocity of the companion are shown in Figs. 2 and 3, respectively. Some 

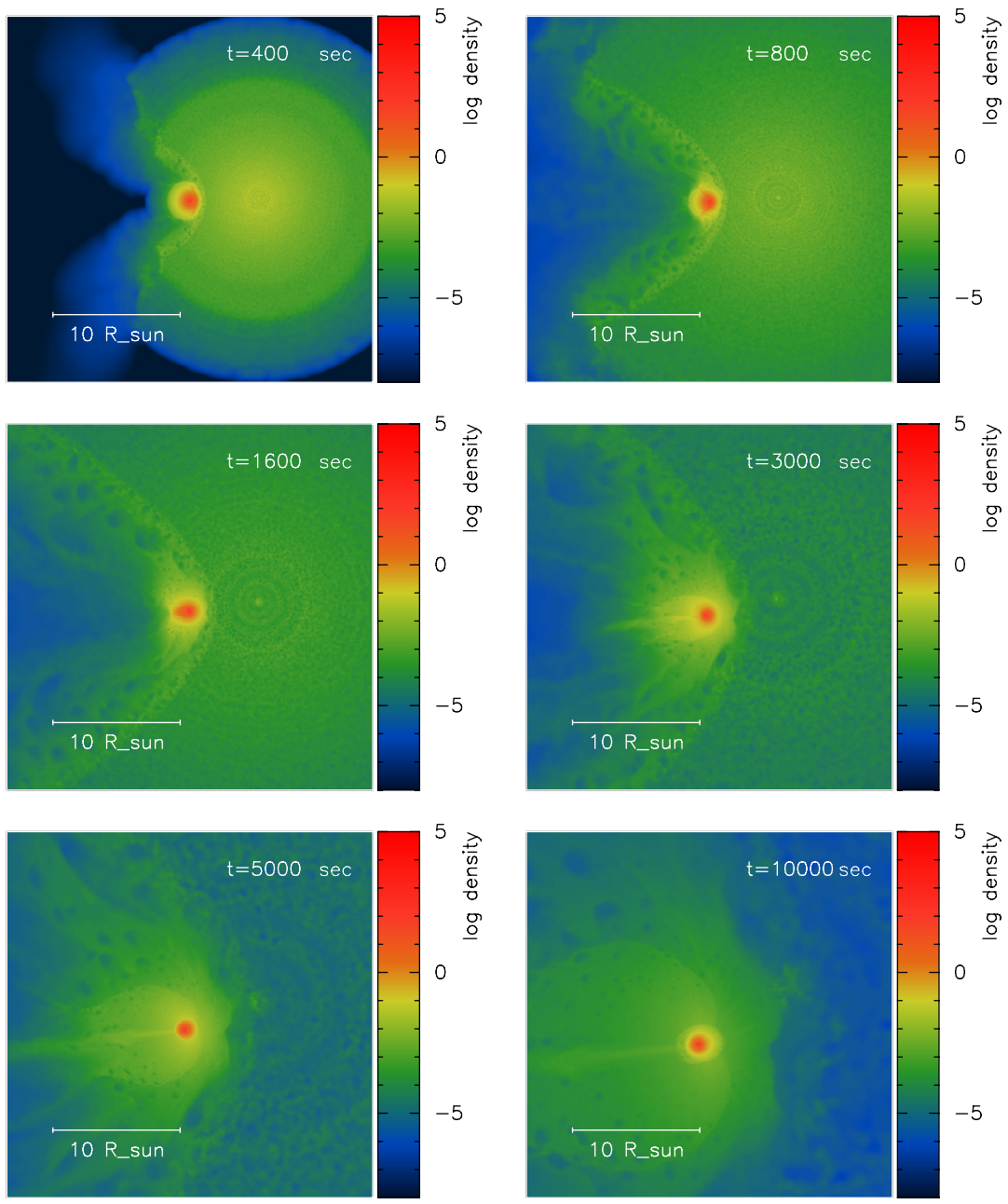

Fig. 3. Same as Fig. 2, but including the orbital motion and the spin of the companion star. The color scale shows the logarithm of the density. Some differences become visible at late times. The plots are made using the freely available SPLASH tool (Price 2007).

small morphological differences between the rotating and nonrotating model are seen due to the symmetry-breaking effects of orbital motion and rotation of the companions star (see Fig. 3).

At the end of the simulation, a mass of $\sim 0.23 M_{\odot}(\sim 19 \%$ of the total companion mass) is stripped from the companion star in the MS_110 model (see Fig. 4). The orbital motion and spin of the companion star only leads to a $\sim 2 \%$ larger unbound mass (see Fig. 4), but basically the same kick velocity $\left(\sim 60 \mathrm{~km} \mathrm{~s}^{-1}\right)$. The result differs from what was reported by Pan et al. (2012b), who found that $16 \%$ more mass can be stripped if the orbital motion and spin is included. In Sect. 5, we again calculate the amount of unbound mass for the model MS_160, which has a higher rotational velocity of $160 \mathrm{~km} \mathrm{~s}^{-1}$, but still only a $4 \%$ difference is found. Moreover, we do not find that the rotation of the companion star significantly affects the post-impact velocity distribution of unbound material and SN Ia ejecta (see Fig. 5). Most of the stripped hydrogen-rich material is confined and hidden close to the center of the SN ejecta. The detection of a hydrogen line may be possible only when the high velocity $\mathrm{SN}$ ejecta becomes transparent.

To summarize, the main results (e.g., the unbound mass, the kick velocity) are similar to our previous work (see Pakmor et al. 2008; Liu et al. 2012) even if the orbital and spin velocity of the secondary are taken into account. This is not surprising, since the orbital and spin velocity of the companion star are obviously much lower than the typical values of the expansion velocity of

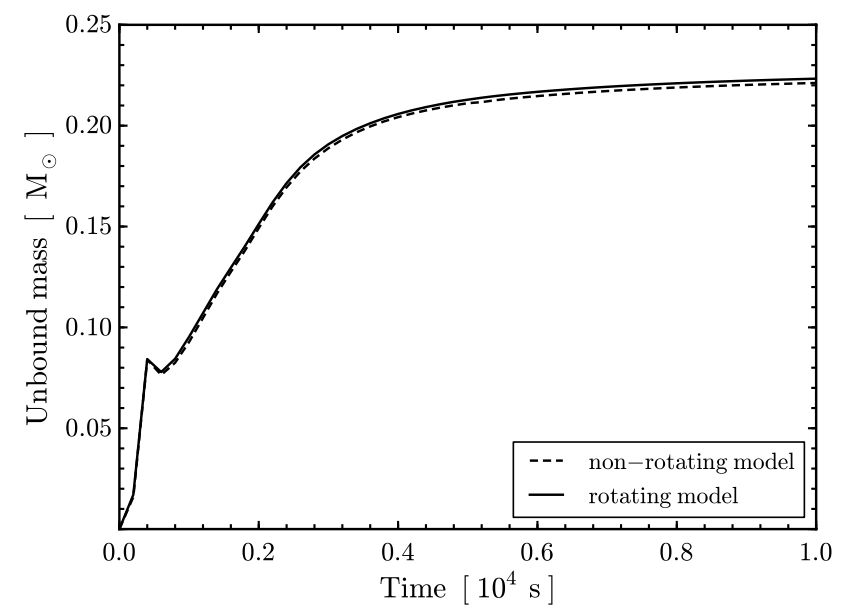

Fig. 4. Unbound mass from the time of explosion with (solid line) and without (dotted line) the orbital rotation and spin of of the companion star for model MS_110.

the SN Ia ejecta $\left(\sim 10^{4} \mathrm{~km} \mathrm{~s}^{-1}\right)$. Therefore, rotation cannot affect the basic physics of interactions between the SN ejecta and the companion star significantly. 

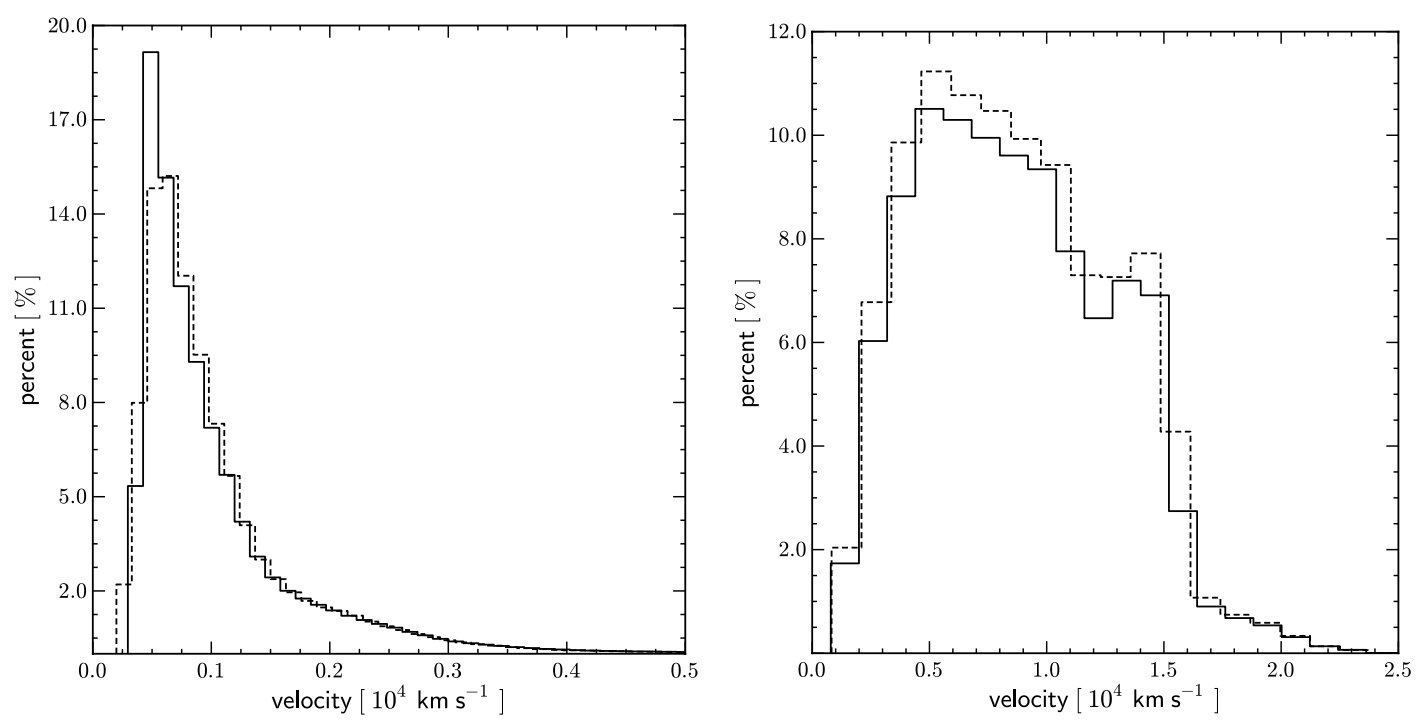

Fig. 5. Velocity distribution of stripped material that originally belonged to the companion star (left figure) and SN ejecta (right figure) for the non-rotating (solid lines) and rotating (dashed lines) MS_110 model.

\section{Post-impact rotation}

In this section, we investigate how the SN Ia explosion affects the rotation of the companion star in our SPH impact simulations by using again the MS_110 model discussed in detail in Sect. 2.2 as a typical case.

\subsection{Temporal evolution of the overall rotation}

At the time of the $\mathrm{SN}$ explosion, the companion star is expected to rotate rapidly at the same frequency as the binary system because of the strong tidal interaction during the RLOF phase. After the SN impact, however, it is uncertain whether it is still possible to use signatures of rapid rotation to single out candidates for donor stars. This depends on the total amount of angular-momentum taken by the stripped material and extreme expansion of the donor star due to SN ejecta heating.

By including the orbital motion and spin of the MS_110 model, we investigate the total angular momentum that can be carried away by the stripped mass, studying the post-impact evolution of rotational velocity of the surviving companion star. During the interaction, the collision of the SN Ia ejecta brings the companion star out of thermal equilibrium. Simultaneously, the spherical symmetry of the companion star is also strongly affected, deforming the shape of the star (the details of density evolution of a remnant star are shown in Sect. 4.2). Therefore, it is difficult to determine the real surface of a surviving companion star and to obtain its overall rotational velocity after the $\mathrm{SN}$ impact. For this purpose, the two following steps were taken to estimate the surface of the surviving companion star in this work.

(a) We divided the surviving star into several hundred spherical shells. The density of the SPH particles in each shell were averaged to calculate a value for that shell. Next, if the density fluctuation in a shell was too strong, it was ignored because the very outer shells are very poorly resolved (SPH particles in these shells are too rare to reasonably resolve the structure of the star). The density profiles of the MS_110 model as a function of explosion time are shown in Fig. 6. At this stage, we obtain the surface of surviving star at $R_{1}$, where the

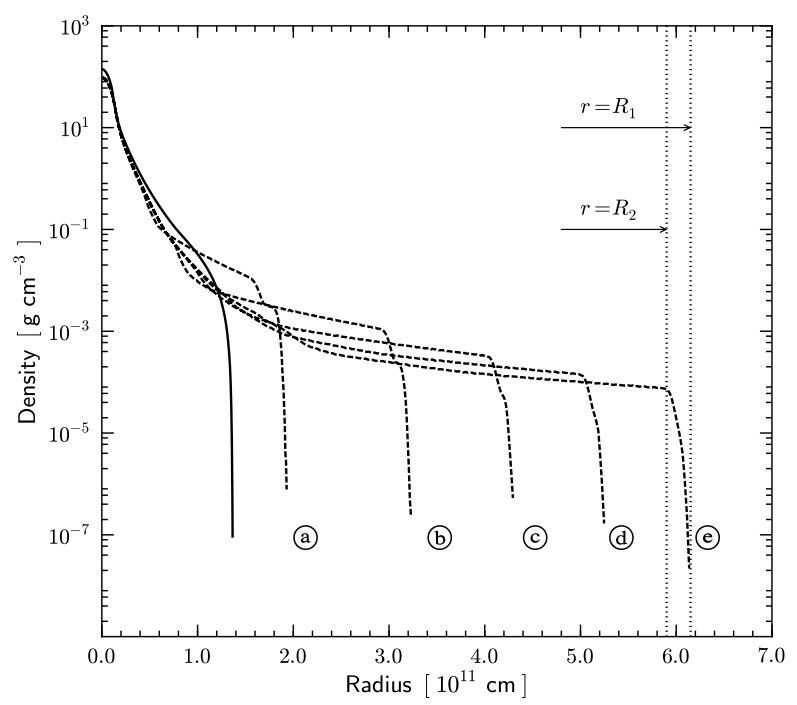

Fig. 6. Density profile of the MS_110 model for different times after the SN explosion. The black solid line corresponds to the initial density profile of the star at the moment of the explosion. All black dashed lines from (a) to (e) show the radial density distribution of the star $(0.4,0.8 \cdots 2.0) \times 10^{4} \mathrm{~s}$ after the impact in time intervals of $4000 \mathrm{~s}$.

tangential velocity with regard to the initial rotation axis, the $z$-axis, is $v_{R_{1}}^{\text {rot }}$.

(b) In Fig. 6, a sharp decrease in density is seen at the outer layer of the surviving companion star. Here, we simply chose the position $R_{2}\left(\sim 95 \%\right.$ of $\left.R_{1}\right)$ of the sharp density jump as the real surface of the star (see the vertical dotted line in Fig. 6). Furthermore, we took the rotation velocity $v_{R_{2}}^{\text {rot }}$ at this surface $R_{2}$ to denote the overall apparent rotational velocity of the surviving companion star $v_{\text {rot }}$ (i.e., equatorial rotational velocity). In Sect. 4.2 we show that the rotational velocity does not change significantly in the outer parts of the stellar envelope. The difference between $v_{R_{1}}^{\text {rot }}$ and $v_{R_{2}}^{\text {rot }}$ is $\$ 5 \%$. On the other hand, it is also difficult to determine the exact position of the photosphere of the surviving companion star. However, Fig. 6 shows that the density at the 

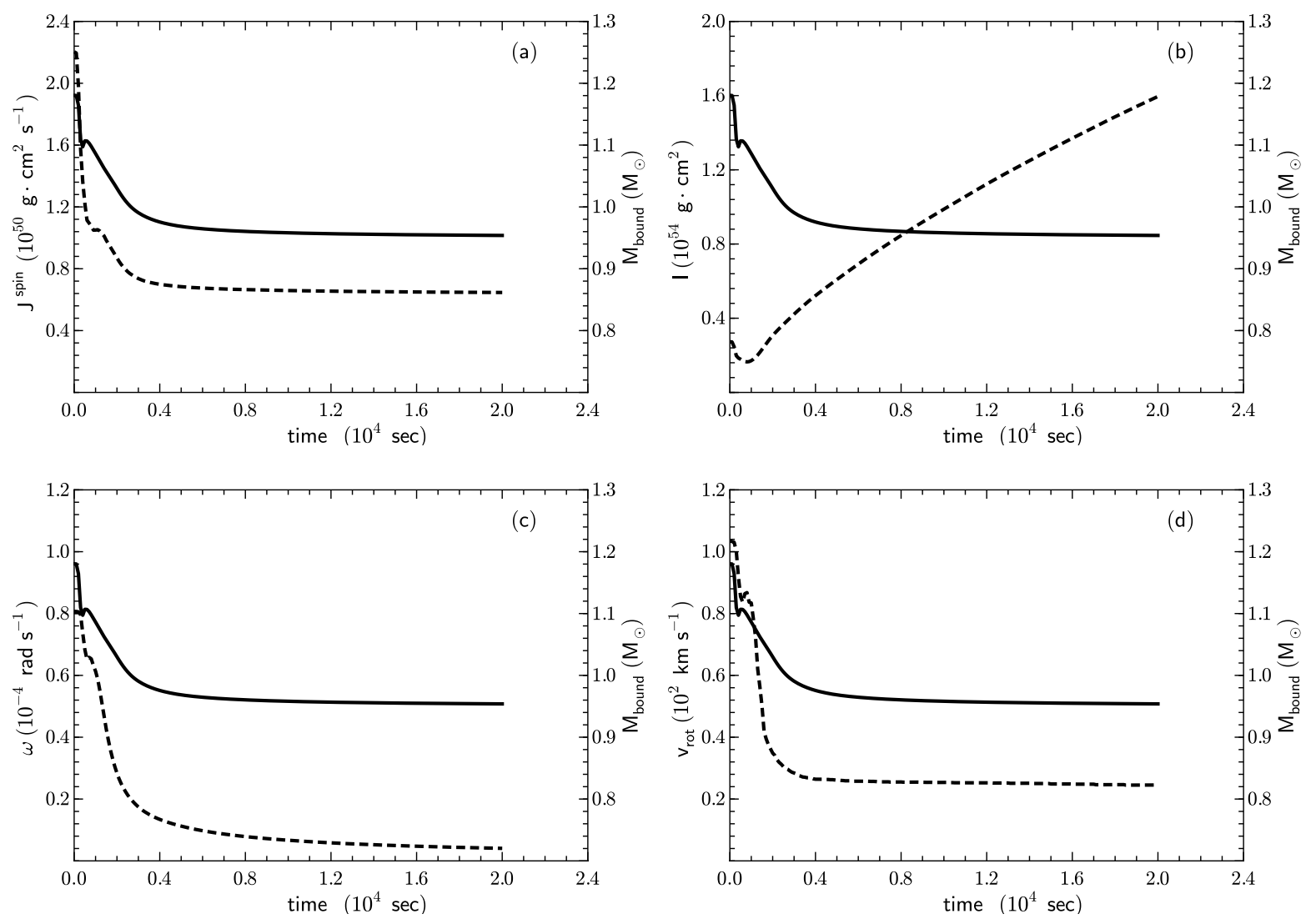

Fig. 7. Temporal evolution of some properties of the companion star after the SN impact in the MS_110 model. The black solid line corresponds to the total bound mass of the companion star $M_{\text {bound }}$. The dashed curves show the temporal changes of the total angular momentum $\left(J^{\text {spin }}\right)$, the moment of inertia $(I)$, the surface angular velocity $(\omega)$, and the surface rotational velocity $\left(v_{\text {rot }}\right)$ of the companion star.

position $R_{2}$ is always higher than the lower density-limit of the photosphere of the sun $\left(\sim 10^{-7} \mathrm{~g} \mathrm{~cm}^{-3}\right)$. Therefore, using the rotational velocity of the star $v_{R_{2}}^{\text {rot }}$ to estimate its observed rotational velocity should be a good approximation.

Figure 7 shows the temporal evolution of $J^{\text {spin }}, I, \omega, v_{\text {rot }}$ and $M_{\text {bound }}$ of the companion star in the impact simulation for the MS_110 model. Here, $J^{\text {spin }}, I, \omega, v_{\text {rot }}$ and $M_{\text {bound }}$ are the total angular momentum, the total moment of inertia, the angular velocity and the rotational velocity at the equator, and the bound mass of the companion star. In the MS_110 model, the companion star has an initial total angular momentum of $2.25 \times 10^{50} \mathrm{~g} \mathrm{~cm}^{2} \mathrm{~s}^{-1}$ at the moment of the explosion. At the end of the simulation, $0.23 M_{\odot}(\sim 19 \%$ of the initial mass $)$ are stripped-off due to the SN impact, carrying a spin angular momentum of $1.63 \times 10^{50} \mathrm{~g} \mathrm{~cm}^{2} \mathrm{~s}^{-1}(\sim 72 \%$ of that before the SN explosion). Therefore, the surface rotational velocity of the star is significantly reduced to $\sim 25 \mathrm{~km} \mathrm{~s}^{-1}$ from an initial rotational velocity of $\sim 110 \mathrm{~km} \mathrm{~s}^{-1}$.

The SN impact takes away both mass and angular momentum, which causes the total angular momentum of the companion star to decrease sharply. Therefore also the angular velocity and the rotation velocity (see the dashed lines in Fig. 7) decrease significantly. Furthermore, the total angular momentum stops decreasing (see dashed line) as the amount of the stripped mass (solid line) reaches a constant value after $5000 \mathrm{~s}$, which was shown in Fig. 7a. As the companion star is puffed up due to energy deposition from the impact of the SN ejecta, its outer layers expand, remarkably increasing the moment of inertia of the star (see Fig. 7b). This explains why the angular velocity continue to decrease slowly after $5000 \mathrm{~s}$ (see dashed line in Fig. 7c) although there is no additional mass-loss and angular-momentum-loss at this moment. However, this expansion does not reduce the rotational velocity of the companion significantly, as is shown by the dashed line in Fig. 7d.

\subsection{Post-impact radial-velocity distribution in the companion star}

In our simulations, the initial rotation of the companion star was set up as a rigid-body rotation, the rotation axis being the $z$-axis. At the moment of the SN explosion, the companion star is spherically symmetric, having the same angular velocity from the center to the surface. Therefore, the rotational velocity increases linearly with radius (see right diagram on the top row of Fig. 8).

Figure 8 shows the density distribution (left column) and the radial profiles of the angular velocity (solid curve) $\omega$ and the rotational velocity (dashed curve) $v_{\text {rot }}$ of the surviving companion star at different times after the explosion for the MS_110 model. After the SN impact, heating by the SN ejecta puffs up the companion star, causing its envelope to expand considerably. This produces an object with a compact inner core and a low-density outer layer (see left column in Fig. 8). The star starts to relax toward a spherical state about $2 \times 10^{4} \mathrm{~s}$ after the impact. However, the density in the outer layers varies by as much as a factor of $\sim 3$ in different directions (see bottom row in Fig. 8), which was also seen in previous simulations (see Marietta et al. 2000; Pakmor et al. 2008; Liu et al. 2012). At this moment, however, the sound crossing time is still longer than the time we follow 

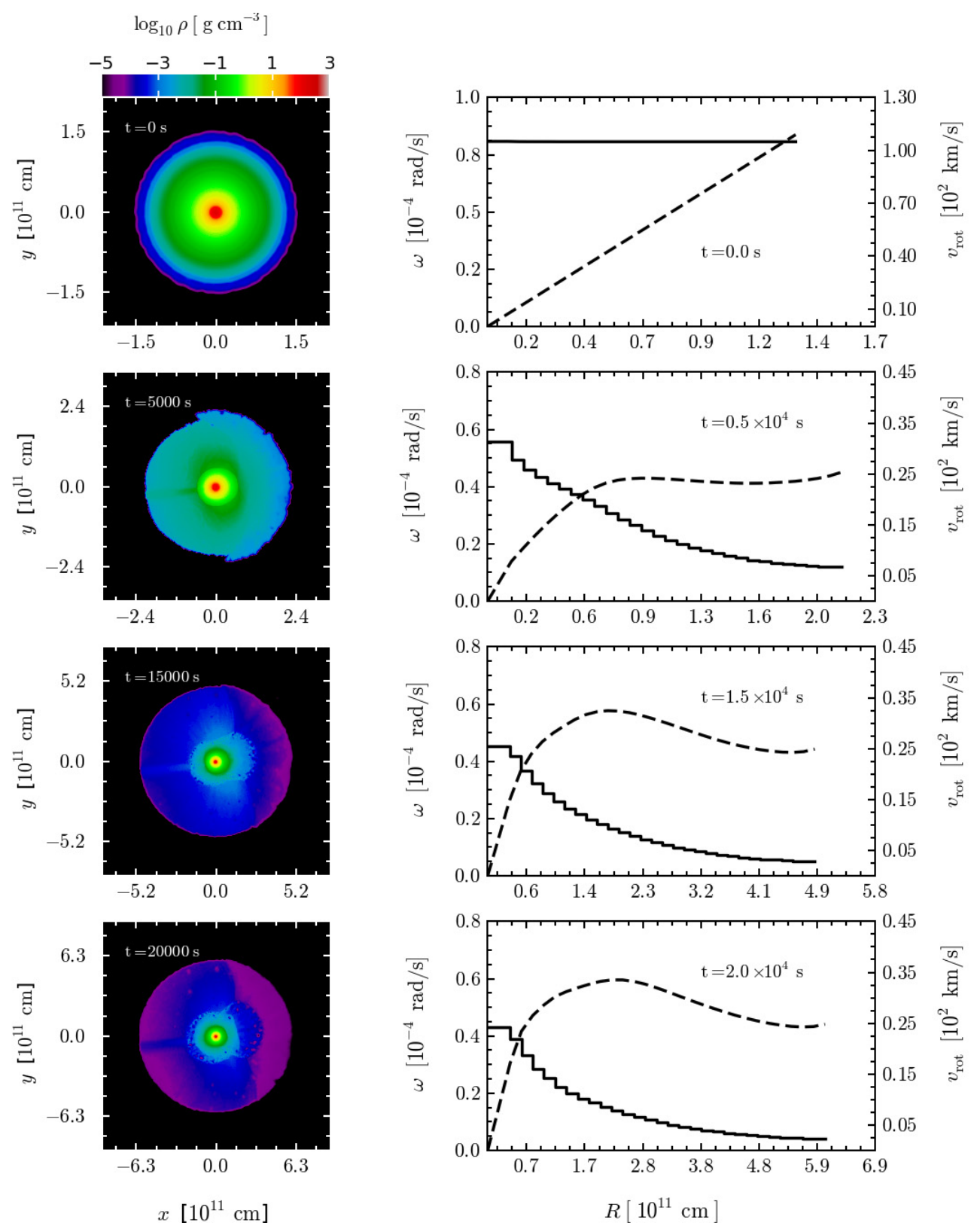

Fig. 8. Left column: temporal evolution of the density distribution of the surviving companion star in model MS_110. Right column: temporal evolution of the radial configurations of the angular velocity $\omega$ (solid line) and the rotational velocity $v_{\text {rot }}$ (dashed line). $R$ is the distance from the rotation axes, the $z$-axis. We use different length scales in different diagrams.

in the simulations $(\sim 5.6 \mathrm{~h})$. Therefore, the star does not reach a spherical state.

The right column of Fig. 8 shows that the equatorial rotational velocity of the companion star drops to $\sim 25 \mathrm{~km} \mathrm{~s}^{-1}$ from the original rotational velocity of $110 \mathrm{~km} \mathrm{~s}^{-1}$ due to the extreme expansion of the star and the angular momentum losses. The angular velocity near the center of the star also decreases below its initial value of $0.8 \times 10^{-4} \mathrm{rad} \mathrm{s}^{-1}$ due to the shock effect that runs through the center, but it becomes stable at late times (see Fig. 9). Additionally, Fig. 8 shows how the radial rotation profiles of the companion star are affected during the collision with the SN Ia ejecta (see also Fig. 9). After the SN impact, the companion star is no longer in rigid-body rotation and its outer layers exhibit some features of differential rotation. At the equator the companion star rotates at a different angular velocity than at higher latitudes. The detailed radial distribution of various rotational properties of model MS_110 $2 \times 10^{4} \mathrm{~s}$ after the explosion are shown in Fig. 10. The angular velocity decreases with increasing latitude of the surviving companion star: the equator rotates with a period of $\sim 14$ days, near the poles it is as short as 2 days. To ensure that changes of the rotational profile of the companion star are purely caused by the impact of the SN explosion, we also made a test run without the SN. It shows that without the SN the rotational velocity profile of a companion star does not change at all over the time of the simulation $\left(2 \times 10^{4} \mathrm{~s}\right)$. In our simulation, we used the rotational velocity at the surface of the post-impact remnant star as the potentially observable velocity. If the photosphere of star were located at a smaller radius rather than the surface, the rotational velocity would be a little bit higher than $\sim 25 \mathrm{~km} \mathrm{~s}^{-1}$. However, the differences of the rotational velocity are not significant for a wide range of outer layers (see Figs. 8 and 9). We also set up the initial rotation of the companion star as rigid rotation, which might be inconsistent with the realistic rotation of a star. A more realistic rotation profile of the star is 


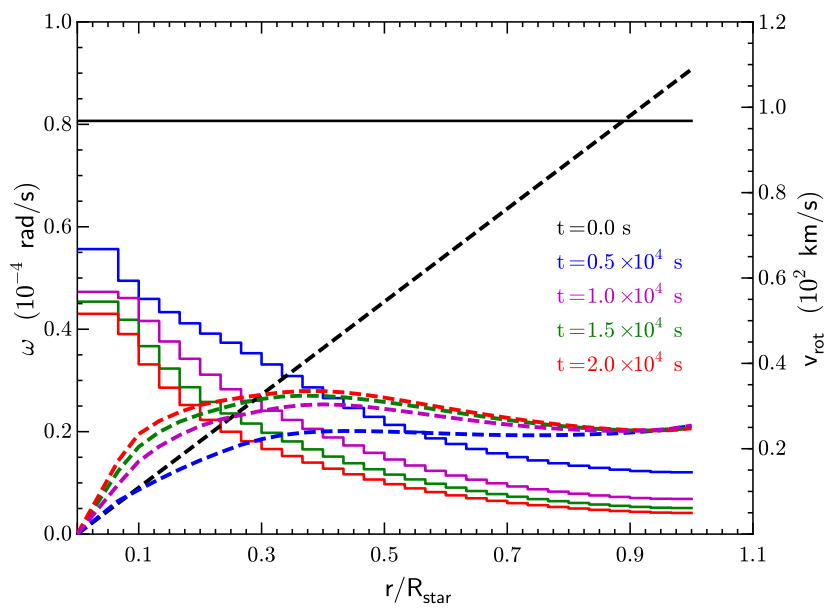

Fig. 9. Radial profiles of angular velocity (solid curves) and rotational velocity (dashed curves) of the companion star in the MS_110 model at different times after the impact $\left(0.0,0.5 \cdots 2.0 \times 10^{4} \mathrm{~s}\right)$.

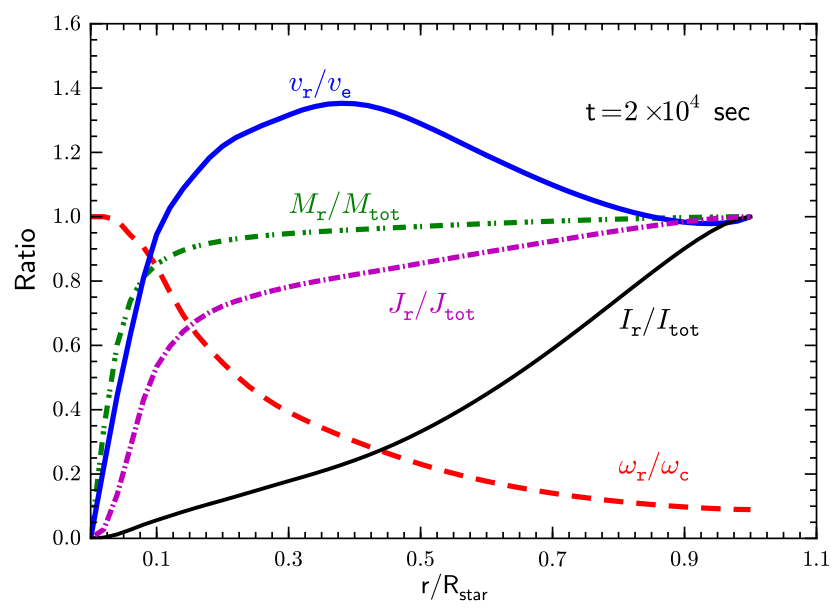

Fig. 10. Radial rotation profiles of the MS 110 model $2 \times 10^{4} \mathrm{~s}$ after the explosion. $R_{\text {star }}$ is the equatorial surface radius and $v_{\mathrm{e}}$ is the equatorial rotational velocity. $M_{r}, J_{r}, I_{r}$ and $\omega_{r}$ are the total bound mass, the total angular momentum, the angular velocity and the moment of inertia of all bound particles interior to the corresponding cylindrical surface $r$. $v_{r}$ is the circular velocity at distance $r$ from the rotation axis. $\omega_{\mathrm{c}}$ is the angular velocity on the rotation axes ( $z$-axis).

required to replace the rigid rotation used here in forthcoming investigations, although we do not expect this to change our main results.

Figure 11 shows the temporal evolution of the radial distribution of the angular momentum of the companion star in model MS_110. The different colors belong to different times since the SN explosion. Each colored solid line shows the details of the radial profile of the angular momentum at that given time. The angular momentum $J_{r}$ was calculated by summing up the total angular momentum of all bound particles interior to the corresponding cylindrical surface at radius $r$. The dashed-dotted line shows the change in total angular momentum as time progresses. Most of the angular momentum of the surviving companion star is concentrated in a small inner core. Outside this core, however, only a small fraction of the total angular momentum is distributed across an extended outer layer (see Fig. 10).

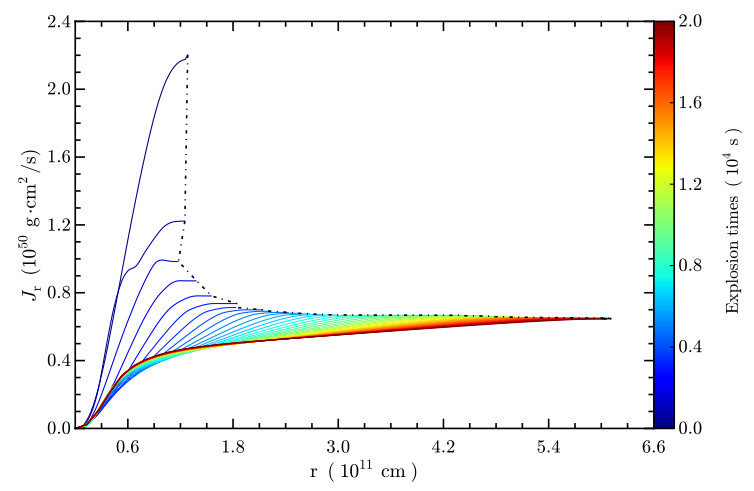

Fig. 11. Evolution of the angular momentum distribution inside the companion star of model MS_110. The color scale gives the time since the explosion. Here, $r$ is the distance from the rotation axes ( $z$-axis), $J_{r}$ denotes the angular momentum of all bound particles interior to the corresponding cylindrical surface $r$. Each colored solid curve shows the radial distribution of the angular momentum $J_{r}$ at a given time. The dashed-dotted curve corresponds to the temporal evolution of the total angular momentum of the companion star.

\section{Comparison with Tycho G}

Tycho's SN (SN 1572) is one of only three historical SNe Ia observed in our galaxy. SN 1572 has the advantage that the SNR field is not so crowded with stars and therefore it provides a good opportunity to observationally identify the companion in case of an SD progenitor. Here, we examine the viability of the candidate Tycho $G$ as the possible surviving companion star in SN 1572 by comparing its observed rotational velocity of $\sim 6 \pm 1.5 \mathrm{~km} \mathrm{~s}^{-1}$ (Kerzendorf et al. 2009, 2012) with the results of our hydrodynamical simulations.

In our MS_110 model, the rotational velocity of the star after the impact is significantly reduced to $23 \%$ of that before the explosion of $\sim 110 \mathrm{~km} \mathrm{~s}^{-1}$ (see Table 1). In Sect. 4.1 we discussed that the surface spin of the companion star is roughly constant from $5000 \mathrm{~s}$ after the explosion. However, we stopped our simulation $2 \times 10^{4} \mathrm{~s}$ after the explosion because of the high computational costs. Podsiadlowski (2003) followed the post-impact evolution of the surviving companion star further and showed that the star might reestablish thermal equilibrium $\sim 10^{3} \mathrm{yr}$ after the explosion (see also Pan et al. 2012a). Figure 9 shows that the radial distribution of the rotational velocity of the surviving companion becomes approximately constant $\sim 1 \times 10^{4} \mathrm{~s}$ after the SN explosion, and the surface velocity at the equator is converged at the end of our hydrodynamics simulations. Therefore, we can safely assume that the surface velocity at the equator of the post-impact companion star would keep a stable value until its thermal equilibrium is established after a few thousand years. Considering that the Tycho SN remnant is only 439 years old, the spin of the MS_110 model $\left(\sim 25 \mathrm{~km} \mathrm{~s}^{-1}\right)$ after the impact is far higher than the observational rotational velocity of the star Tycho G $\left(6 \pm 1.5 \mathrm{~km} \mathrm{~s}^{-1}\right.$ according to Kerzendorf et al. 2009, 2012).

Han (2008) carried out detailed binary evolution calculations for the WD+MS channel of SNe Ia, in which RLOF starts when the companion star is on the MS or in the Hertzsprung-gap phase. They obtained many properties of the companion star at the moment of the SN explosion (e.g. their masses, spatial velocity, effective temperature, luminosity, surface gravity, etc). These properties might be verified by the observations. The distribution of properties of companion stars in the plane of $\left(v_{\mathrm{rot}}^{\mathrm{SN}}, M_{2}^{\mathrm{SN}}\right)$ from Han (2008) is shown in Fig. 12a. Based on the observations of 

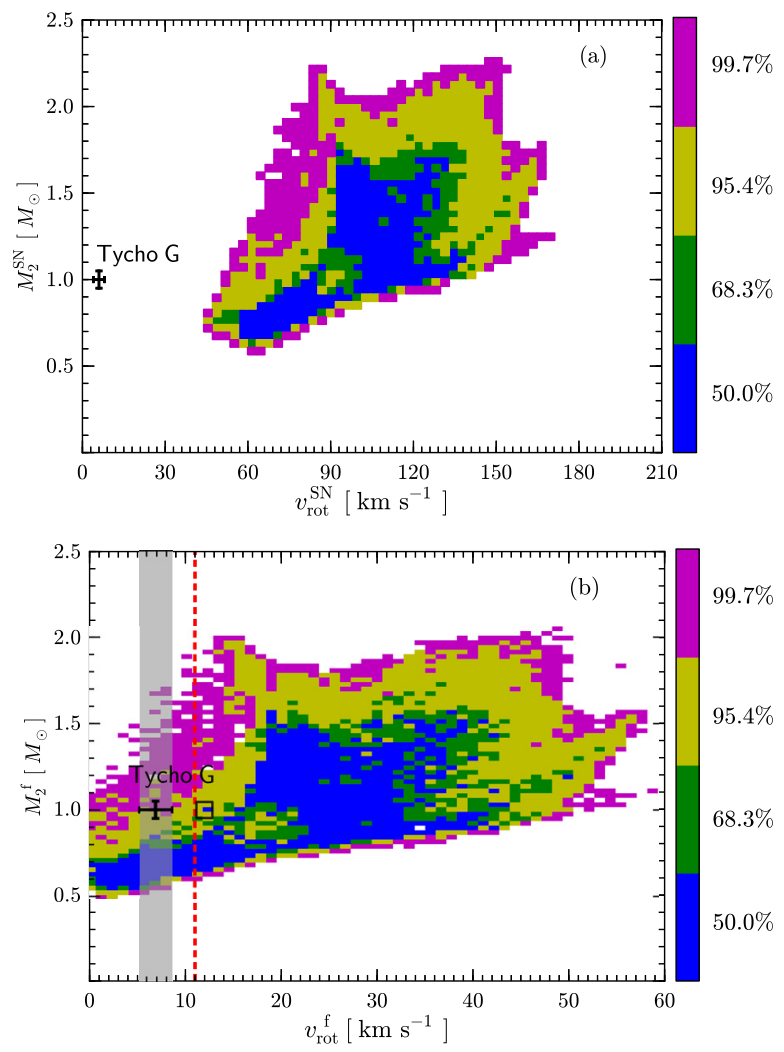

Fig. 12. Panel a) distribution of rotation velocity versus mass of the companion stars at the moment of the explosion as obtained by Han (2008). The number of systems decreases from the inner to outer regions. The blue area contains $50.0 \%$ of all stars, blue plus green $68.3 \%$, and so on. Panel b) as panel a), but now the distribution is shown after the impact. The distribution is shifted to lower rotational velocity and slightly lower masses. The unbound mass stripped by the SN impact is calculated by using Eq. (2) of Liu et al. (2012), and the post-impact rotational velocity of the companion star is computed employing the linear relation shown in Fig. 13 (see text). The location of Tycho G according to Kerzendorf et al. $(2009,2012)$ is shown with the error in black assuming a solar mass star with 5\% error (Ruiz-Lapuente et al. 2004) and an inclination angle of $i=60^{\circ}$. The black square gives the location of Tycho $\mathrm{G}$ for an assumed inclination angle of $i=30^{\circ}$. The red vertical dashed line corresponds to the lowest rotational velocity covered by our hydrodynamical simulations (i.e., MS_081 model in Table 1).

Kerzendorf et al. (2009, 2012), the location of the rotation of the star Tycho $\mathrm{G}$ is shown with black error bars. Clearly, because of the bound rotation before the impact, Tycho $G$ is far away from the allowed region.

Next we estimated the rotational velocity of the stars shown in Fig. 12a after the impact. To obtain the distribution of rotational velocities of surviving companions after the SN Ia explosion, we re-performed 1D consistent binary evolution calculations to construct the structures of the companions, obtaining additional three consistent models named MS_081, MS_131 and MS_160 with different rotational velocities of $81 \mathrm{~km} \mathrm{~s}^{-1}$, $131 \mathrm{~km} \mathrm{~s}^{-1}$ and $160 \mathrm{~km} \mathrm{~s}^{-1}$ (see Table 1). We then used these three models as input into our impact simulations to investigate the dependence of the rotational velocity of companion star after the impact on its value before the explosion. The properties of all companion models are shown in Table 1 . We find that to a good approximation the post-impact rotational velocity of star scales linearly with the pre-explosion velocity, as is shown in Fig. 13, and can be fitted by

$V_{\text {rot }}^{\mathrm{f}}=0.52 \cdot V_{\text {rot }}^{\mathrm{SN}}-29.8\left(\mathrm{~km} \mathrm{~s}^{-1}\right)$,

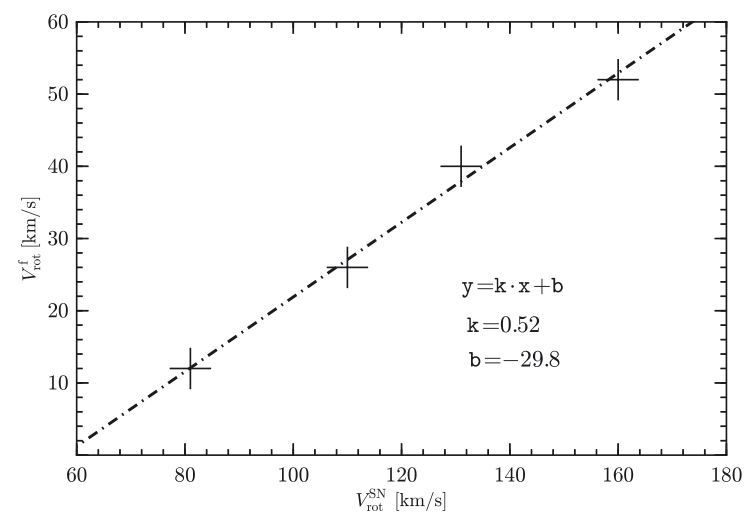

Fig. 13. Initial rotational velocity at the moment of the $\mathrm{SN}$ explosion $V_{\text {rot }}^{\mathrm{SN}}$ versus rotational velocity after the impact $V_{\text {rot }}^{\mathrm{f}}$ for four different MS companion star models (see Table 1).

where $V_{\text {rot }}^{\mathrm{f}}$ is the post-impact rotational velocity at the end of the simulations, and $V_{\text {rot }}^{\mathrm{SN}}$ corresponds to the star's initial rotational velocity at the moment of the explosion.

Previously, Liu et al. (2012) found that the unbound mass of the companion star caused by the SN impact is strongly dependent on the ratio of separation to the radius of the companion star, $a_{\mathrm{f}} / R_{2}$. This relation can be fitted by a power law if the effect of the different structures of the companion stars is neglected (see also Marietta et al. 2000; Pakmor et al. 2008; Pan et al. 2012b):

$M_{\text {stripped }}=C_{1}\left(\frac{a_{\mathrm{f}}}{R_{2}}\right)^{\beta} M_{\odot}$,

where $C_{1}$ is a fitting constant that depends on the different companion star models. The parameter $\beta$ is the corresponding powerlaw index.

Therefore, adopting the power-law relation (see also Eq. (2) of Liu et al. 2012) and the linear relation (2) obtained from the data shown in Fig. 13, we calculated the final bound mass and the post-impact rotational velocity of surviving companion stars based on all companion models from the population synthesis study of Han (2008). The results are presented in Fig. 12b. The location of Tycho $G$ is also shown with a black error bars. Here, we assumed that Tycho $\mathrm{G}$ is a one-solar-mass star (Ruiz-Lapuente et al. 2004) and its rotational velocity is $6 \pm 1.5 \mathrm{~km} \mathrm{~s}^{-1}$ (Kerzendorf et al. 2012). Figure 12 shows that Tycho $\mathrm{G}$ is located in the outer region of $95.4 \%$ of all systems, which casts doubts on Tycho $\mathrm{G}$ star as a promising candidate in SD progenitors of SNe Ia.

However, it is not possible to exclude the star Tycho G completely for several reasons.

1. The errors shown in Fig. 12 are based on an assumption that Tycho $\mathrm{G}$ is a one-solar-mass star (Ruiz-Lapuente et al. 2004). However, it is very difficult to determine the actual mass from the observations. Therefore, Tycho $G$ should be located in the vertical gray strip at its given rotation velocity in Fig. 12 b. If Tycho $G$ is a $0.6-0.7 M_{\odot}$ star rather a solar mass star, it could more likely be a candidate for the companion star of SN 1572 in the SD scenario. Moreover, the errors given in Fig. 12 are based on the assumption that the observed rotational velocity of Tycho $\mathrm{G}$ is reduced by an inclination angle of $60^{\circ}$. However, if the inclination angle is $30^{\circ}$ instead (see black square in Fig. 12b), the true rotational velocity of Tycho $\mathrm{G}$ would be higher, thus supporting the interpretation that it is the surviving companion of SN 1572. 
Figure 14 shows the distribution of the observable $v_{\text {rot }}^{\mathrm{f}} \sin i$ assuming a random viewing angle.

2. In our simulations, we found that the post-impact rotation velocity of the surviving companions is linearly dependent on the initial rotational velocity at the moment of the explosion (see Fig. 13). However, we restricted our simulations to cover a range of initial rotational velocities of only $80 \mathrm{~km} \mathrm{~s}^{-1}<v_{\text {rot }}^{\mathrm{SN}}<160 \mathrm{~km} \mathrm{~s}^{-1}$ (which corresponds to the range of the post-impact rotational velocities of $10 \mathrm{~km} \mathrm{~s}^{-1}<$ $v_{\text {rot }}^{\mathrm{f}}<50 \mathrm{~km} \mathrm{~s}^{-1}$ ) because of computational costs. The companion star models with lower initial rotation velocity always evolve to the giant phase with larger orbital separation at the time when the supernova explodes, which significantly increases the computational cost of the simulations. Figure 12a shows that some companion-star models $(\sim 14 \%$ of all binaries) are located in a range of $v_{\text {rot }}^{\mathrm{SN}}<80 \mathrm{~km} \mathrm{~s}^{-1}$ (i.e., regions left of the red vertical dashed line in Fig. 12b). These models may not obey the linear relation as shown in Fig. 13, but rather follow something close to a power law relation. Therefore, the models with post-impact rotational velocities close to zero in Fig. 12b might not be realistic. However, $\sim 86 \%$ of all models are located in the range that we followed in the simulations $\left(80 \mathrm{~km} \mathrm{~s}^{-1}\right.$ to $\left.160 \mathrm{~km} \mathrm{~s}^{-1}\right)$, indicating that most of post-impact surviving companion stars in the WD+MS scenario should be rapidly rotating after the impact.

3. The post-impact masses of the companion stars shown in Fig. 12b were calculated by directly adopting the power law relation from Eq. (2) of Liu et al. (2012), which ignores the effect of the different companion structures. However, Liu et al. (2012) found that the details of the companion structure also play an important role in determining the amount of unbound mass caused by the impact. Nonetheless, the ratio of separation to the radius of companion star $a_{\mathrm{f}} / R_{2}$ is the most important parameter to determine the unbound mass (Liu et al. 2012).

4. We assumed that the surviving companion star has a constant surface rotational velocity after $\sim 5000 \mathrm{~s}$ after the SN Ia impact. However, we only carried out our simulations to $2 \times 10^{4} \mathrm{~s}$ after the explosion. Extended calculations based on the results of our impact simulations are still required to follow in detail the post-impact rotation of the star during its re-equilibration phase. For instance, very recently Pan et al. (2012a) showed that the remnant star after the SN impact could continue to expand on a local thermal timescale of $\sim 10^{3}$ yr before it shrinks again.

\section{Rotation velocity after re-equilibration}

After the supernova impact, the companion star puffs up and the stellar envelope is out of thermal equilibrium. The postimpact remnant star will recover the state of thermal equilibrium on the Kelvin-Helmholtz timescale of $\sim 10^{3}-10^{5}$ yrs (Pan et al. 2012a; Marietta et al. 2000; Podsiadlowski 2003). To estimate the rotation rate after thermal equilibrium is reestablished, we made the simplifying assumption that the remnant star has a constant rotational velocity during the re-equilibration phase. After thermal equilibrium is established, the post-impact remnant star will shrink and return to the state of an MS-like star. As a result, the surface rotational velocity of the remnant star will increase again, and we assumed that the angular momentum of the star would redistribute towards rigid-body rotation. If the angular momentum is given roughly by $J=\alpha M R^{2} \omega$ before the

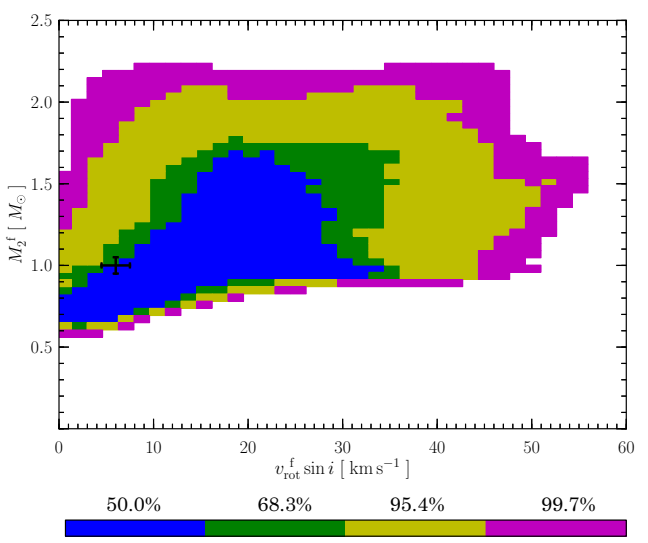

Fig. 14. Same as Fig. 12b, but showing the observable quantity $v_{\text {rot }}^{\mathrm{f}} \sin i$, where $i$ is the inclination angle. The distribution of $i$ is chosen corresponding to a random viewing angle. The black cross is again the location of Tycho $\mathrm{G}$ with an observed rotation velocity of $6 \pm 1.5 \mathrm{~km} \mathrm{~s}^{-1}$.
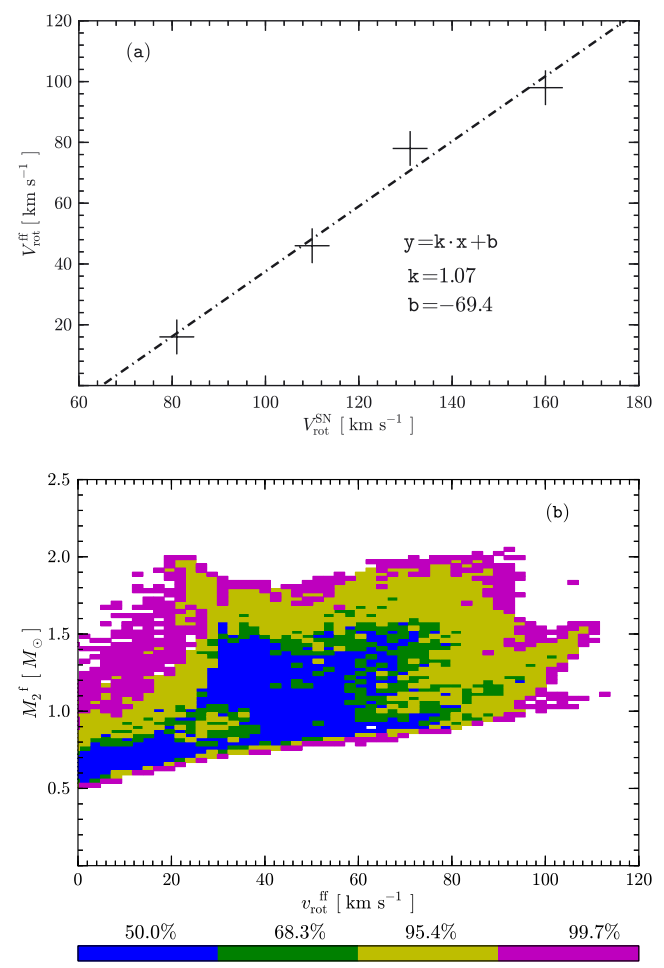

Fig. 15. Top panel: similar to Fig. 13, but in the plane of $\left(V_{\mathrm{rot}}^{\mathrm{SN}}, V_{\mathrm{rot}}^{\mathrm{ff}}\right)$. Note that $V_{\text {rot }}^{\mathrm{ff}}$ is the rotation velocity of the remnant star after thermal equilibrium is reestablished. Bottom panel: similar to Fig. 12b, but in the plane of $\left(V_{\mathrm{rot}}^{\mathrm{ff}}, M_{2}^{\mathrm{f}}\right)$.

SN explosion and after re-equilibration, and also has a constant value of the parameter $\alpha$, the rotational velocity $\left(V_{\text {rot }}^{\mathrm{ff}}\right)$ of the remnant star after the re-equilibration can be determined once the radius of the re-equilibration star $R$ is fixed. To estimate the final radius of the remnant star, the MS relation $R \propto M^{2 / 3}$ was used (see also Marietta et al. 2000). By adopting the post-impact angular momentum $J_{\text {spin }}^{\mathrm{f}}$ and the value of $\alpha$ that was calculated for the pre-SN companion star model, we can estimate the rotational velocity of the remnant star after the thermal equilibrium is reestablished. The rotational velocity of different companionstar models before the explosion and after the re-equilibration can also be fitted with a linear relation in good approximation (see Fig. 15a). In Fig. 15b we also show the distribution 
of the rotational velocities $V_{\text {rot }}^{\mathrm{ff}}$ after re-equilibration, employing the same method as in Sect. 5. It can be seen that the companion stars relax to higher rotation velocity again after the thermal equilibrium is re-established (at least about a few thousand years after the SN explosion).

\section{Summary and conclusions}

We have modeled the impact of SN Ia ejecta on their MS companion stars by means of 3D SPH hydrodynamical simulations that included the orbital and spin velocity of the companion stars. The MS companion stars were constructed by using Eggleton's 1D stellar evolution code making use of the optically thick wind model of Hachisu et al. (1999). We found that the orbital motion and the spin of the companion star do not significantly affect the amount of unbound mass and the kick velocity caused by the SN impact. This result, obtained with an SPH code, differs from what was found previously by Pan et al. (2012b), who from their grid-based FLASH code simulations concluded that these two properties increase the amount of unbound mass by up to $\sim 16 \%$, but also found that the kick velocity is not affected. We have shown that the $\mathrm{SN}$ impact affects the rotation rate of the companion stars and their rotation laws. In our simulations we found that the $\mathrm{SN}$ impact removes as much as $55 \%$ to $89 \%$ of the initial angular momentum because $14 \%$ to $23 \%$ of the initial mass is stripped from the MS companion star. The remnant expands significantly after the impact, which causes the equatorial surface rotational velocity to drop significantly to $14 \%$ to $32 \%$ of the original value. Additionally, we found that the post-impact rotational velocities of companion stars depend linearly on those prior to the explosion. Compared with the observed rotational velocity of the presumed companion star of Tycho's supernova, Tycho G, the final rotational velocity we obtain in our simulations is still higher by at least a factor of two. Whether this difference is significant and may cast doubts on the suggestion that Tycho G is the companion of SN 1572 has to be investigated in future studies. In particular, a more accurate mass and inclination of Tycho $G$ would help, as well as simulations that follow the evolution for much longer than we can do with our explicit SPH code. Furthermore, by using the population synthesis results of Han (2008), we showed the distributions of the rotational velocities of surviving companions after the impact, which may be very useful for future observations to identify the surviving companion stars in SNRs.

Acknowledgements. Z.W.L. and Z.W.H. thank the financial support from the MPG-CAS Joint Doctoral Promotion Program (DP) and Max Planck Institute for Astrophysics (MPA). This work is supported by the National Basic Research Program of China (Grant No. 2009CB824800), the National Natural Science Foundation of China (Grant Nos. 11033008 and 11103072) and the Chinese Academy of Sciences (Grant N0. JCS2-WY-T24). The work of F.K.R. was supported by Deutsche Forschungsgemeinschaft via the Emmy Noether Program (RO 3676/1-1) and by the ARCHES prize of the German Federal Ministry of Education and Research (BMBF). The simulations were carried out at the Computing Center of the Max Planck Society, Garching, Germany.

\section{References}

Alexander, D. R., \& Ferguson, J. W. 1994, ApJ, 437, 879

Chen, X.-F., \& Tout, C. A. 2007, Chin. J. Astron. Astrophys., 7, 245

Dilday, B., Howell, D. A., Cenko, S. B., et al. 2012, Science, 337, 942

Eggleton, P. P. 1971, MNRAS, 151, 351

Eggleton, P. P. 1972, MNRAS, 156, 361

Eggleton, P. P. 1973, MNRAS, 163, 279

Foley, R. J., Kromer, M., Howie Marion, G., et al. 2012, ApJ, 753, L5 Fuhrmann, K. 2005, MNRAS, 359, L35

González Hernández, J. I., Ruiz-Lapuente, P., Filippenko, A. V., et al. 2009, ApJ, 691,1

Hachisu, I., Kato, M., Nomoto, K., \& Umeda, H. 1999, ApJ, 519, 314

Hachisu, I., Kato, M., Kato, T., \& Matsumoto, K. 2000, ApJ, 528, L97

Hachisu, I., Kato, M., \& Nomoto, K. 2008, ApJ, 679, 1390

Han, Z. 2008, ApJ, 677, L109

Han, Z., \& Podsiadlowski, P. 2004, MNRAS, 350, 1301

Hillebrandt, W., \& Niemeyer, J. C. 2000, ARA\&A, 38, 191

Howell, D. A. 2011, Nature Commun., 2, 350

Hoyle, F., \& Fowler, W. A. 1960, ApJ, 132, 565

Iben, Jr., I., \& Tutukov, A. V. 1984, ApJS, 54, 335

Iglesias, C. A., \& Rogers, F. J. 1996, ApJ, 464, 943

Ihara, Y., Ozaki, J., Doi, M., et al. 2007, PASJ, 59, 811

Kerzendorf, W. E., Schmidt, B. P., Asplund, M., et al. 2009, ApJ, 701, 1665

Kerzendorf, W. E., Yong, D., Schmidt, B. P., et al. 2012, ApJ, submitted [arXiv: 1210.2713]

Leibundgut, B. 2008, General Relativity and Gravitation, 40, 221

Leonard, D. C. 2007, ApJ, 670, 1275

Liu, Z. W., Pakmor, R., Röpke, F. K., et al. 2012, A\&A, 548, A2

Marietta, E., Burrows, A., \& Fryxell, B. 2000, ApJS, 128, 615

Meng, X., \& Yang, W. 2010, ApJ, 710, 1310

Nomoto, K., Thielemann, F.-K., \& Yokoi, K. 1984, ApJ, 286, 644

Nomoto, K., Iwamoto, K., \& Kishimoto, N. 1997, Science, 276, 1378

Pakmor, R., Röpke, F. K., Weiss, A., \& Hillebrandt, W. 2008, A\&A, 489, 943

Pakmor, R., Kromer, M., Röpke, F. K., et al. 2010, Nature, 463, 61

Pakmor, R., Hachinger, S., Röpke, F. K., \& Hillebrandt, W. 2011, A\&A, 528, A117

Pakmor, R., Edelmann, P., Röpke, F. K., \& Hillebrandt, W. 2012a, MNRAS, 424, 2222

Pakmor, R., Kromer, M., Taubenberger, S., et al. 2012b, ApJ, 747, L10

Pan, K.-C., Ricker, P. M., \& Taam, R. E. 2010, ApJ, 715, 78

Pan, K.-C., Ricker, P. M., \& Taam, R. E. 2012a, ApJ, 760, 21

Pan, K.-C., Ricker, P. M., \& Taam, R. E. 2012b, ApJ, 750, 151

Patat, F., Chandra, P., Chevalier, R., et al. 2007, Science, 317, 924

Perlmutter, S., Aldering, G., Goldhaber, G., et al. 1999, ApJ, 517, 565

Phillips, M. M. 1993, ApJ, 413, L105

Phillips, M. M., Lira, P., Suntzeff, N. B., et al. 1999, AJ, 118, 1766

Podsiadlowski, P. 2003, unpublished [arXiv:astro-ph/0303660]

Pols, O. R., Tout, C. A., Schroder, K.-P., Eggleton, P. P., \& Manners, J. 1997, MNRAS, 289, 869

Price, D. J. 2007, PASA, 24, 159

Ricker, P. M., Pan, K.-C., \& Taam, R. E. 2010, in AIP Conf. Ser. 1314, eds. V. Kologera, \& M. van der Sluys, 250

Riess, A. G., Filippenko, A. V., Challis, P., et al. 1998, AJ, 116, 1009

Ruiz-Lapuente, P., Comeron, F., Méndez, J., et al. 2004, Nature, 431, 1069

Schroder, K.-P., Pols, O. R., \& Eggleton, P. P. 1997, MNRAS, 285, 696

Shappee, B. J., Stanek, K. Z., Pogge, R. W., \& Garnavich, P. M. 2013, ApJ, 762, L5

Springel, V. 2005, MNRAS, 364, 1105

Springel, V., Yoshida, N., \& White, S. D. M. 2001, New Astron., 6, 79

Sternberg, A., Gal-Yam, A., Simon, J. D., et al. 2011, Science, 333, 856

Thoroughgood, T. D., Dhillon, V. S., Littlefair, S. P., Marsh, T. R., \& Smith,

D. A. 2001, MNRAS, 327, 1323

Wang, B., \& Han, Z. 2010a, MNRAS, 404, L84

Wang, B., \& Han, Z. 2010b, A\&A, 515, A88

Wang, B., \& Han, Z. 2012, New Astron. Rev., 56, 122

Webbink, R. F. 1984, ApJ, 277, 355 